\title{
Effects of arousal level on short- and long-term habituation of the orienting response
}

\author{
ROBERT J. GATCHEL and ELIZABETH GAAS \\ University of Texas-Arlington, Room 309, Life Science Building, Arlington, Texas 76019
}

\begin{abstract}
The effects of sympathetic arousal level on short- and long-term habituation of the skin conductance response component of the orienting response (OR) were investigated in an experiment employing 28 subjects. Threat of shock was used to increase the arousal level of one group of subjects. These subjects were compared to a group which was not threatened by shock. Results indicated that threat of shock significantly increased tonic skin conductance level. More importantly, this increase in physiological arousal level was associated with decreased short- and long-term habituation of the OR. These results demonstrate that physiological arousal level significantly affects the habituation process.
\end{abstract}

It has been demonstrated that interstimulus interval (ISI) length differentially affects short- and long-term habituation of autonomic components of the orienting response (OR) in human subjects (Gatchel, 1975). Tone exposure at a short 20-sec ISI length resulted in greater relative habituation than exposure at a longer 100 -sec ISI length. However, when subjects were tested $15 \mathrm{~min}$ later with both 20 and 100 -sec ISIs, habituation was greater for the long-ISI group. Thus, the results indicated that long-term OR habituation was directly related to ISI length while short-term habituation was inversely related to ISI length. A similar effect has been found for startle-response habituation in the rat (Davis, 1970).

Gatchel (1975) suggested that two different inhibitory processes must be active in order to account for the short- and long-term habituation differences. During short-term habituation, the high rate of stimulus input present in the 20 -sec ISI group may have resulted in too brief a time period to have allowed complete recovery of a response to one stimulus before the next succeeding stimulus was presented. It was suggested, therefore, that an autonomic response refractory period may have accounted for the greater short-term habituation demonstrated by the 20 -sec ISI group.

It was also suggested that the long-term habituation differences may have been due to arousal level effects. This suggestion was based on the finding that, relative to the long-ISI group, the short-ISI group was associated with a higher skin-conductance level by the end of the initial short-term habituation phase of the experiment, which was significantly maintained when subjects were tested for long-term habituation 15-min later. It was speculated that the high rate of stimulus input may have generated and maintained

This research was supported in part by a grant to the first author from the University of Texas at Arlington Organized Research Fund. an elevated level of sympathetic arousal in the short-ISI group. This high level of arousal may have interfered with the consolidation process that prompts habituation and may have potentiated responding on subsequent exposures to the test stimulus.

If arousal level does significantly affect the habituation process, then other techniques for eliciting sympathetic activity should similarly interfere with secondary habituation. The present experiment was designed to examine whether elevation of sympathetic arousal, directly elicited by threat of shock, significantly interferes with long-term habituation. These data should more clearly illuminate whether physiological arousal level is an important factor in the habituation process.

\section{METHOD}

\section{Subjects}

Subjects consisted of 28 female undergraduates from the introductory psychology subject pool at the University of Texas at Arlington. They received points to be applied to their course grade for participation in this experiment. All subjects reported having normal hearing.

\section{Apparatus}

Skin resistance was continuously monitored throughout the experiment on a Narco Bio-Systems physiograph. Beckman silver-silver chloride electrodes (1-cm radius) were attached to the palm and ventral side of the wrist of the subject's nonpreferred hand to record skin resistance. An inactive "dummy" shock electrode was attached to the wrist of the subject's preferred hand. Johnson \& Johnson K-Y jelly was used as the electrolyte in recording skin resistance. A physiogrph GSR coupler imposed a current of 10 microA through the two electrodes. Two channels of electrodermal data were collected. The first channel was dc coupled, and it recorded tonic skin-resistance levels at a low sensitivity setting $(2,500$-ohm resistance change sensitivity). The second channel was capacitor coupled, with a 6-sec time constant, which allowed the recording of short-term phasic responses at a high sensitivity setting (500-ohm resistance change sensitivity).

Stimuli were 2-sec tones presented binaurally over a matched set of Grason-Stadler earphones. The tones were generated by a Hewlett-Packard $200 \mathrm{CD}$ oscillator, with a ramp-control electronic switch imposing a 10-msec rise and decay time on the tones. The tone intensity was $65 \mathrm{~dB}$, as measured by scale Cs of a General 
Radio Company sound-level meter. The 2 -sec tone presentation was controlled by a Scientific Prototype interval timer.

\section{Experimental Design and Procedure}

Subjects were randomly assigned to one of two groups (14 subjects per group), which differed only in the instructions that were administered. In Part I of the experiment, all subjects received tones every 50,60 , or $70 \mathrm{sec}$, with a mean ISI of $60 \mathrm{sec}$. Each subject received 15 tones. In Part II of the experiment, which tested for long-term habituation effects, 15 tones were again administered with a mean ISI presentation rate of $60 \mathrm{sec}$

The subject was seated in a comfortable chair in a partially soundproof room. After the recording electrodes were attached, the experimenter calibrated the recording equipment in an adjacent room. The experimenter then read the differential group instructions. Group 1 subjects were administered instructions which informed them that they would hear a series of tones. Group 2 subjects received similar instructions, but with the addition of a threat of shock statement. The instructions are presented below. Group 1 subjects were not read the sentence within the parentheses. This sentence, intended to elicit an elevation of sympathetic arousal, was administered only to the Group 2 subjects. "During the course of the experiment you will be presented some tones through the headset. (Also, you may receive a mild electric shock through the electrode on your wrist at some point during the experiment.) Please sit quietly, relax but do not fall asleep. Please try to move as little as possible since movement interferes with our physiological recording. I will let you know over the intercom when the session is completed. Do you have any questions? If not, we will get started in a few minutes."

After reading the instructions, the experimenter left the room and. after a 15-min baseline period, presented the stimuli. It should be noted that at no time during the experiment was shock ever administered to subjects. At the completion of Part I of the experiment, subjects were read another set of instructions. These instructions, presented below, introduced Part II and were intended to eliminate the threat of shock in the Group 2 subjects. Again, the sentence within the parentheses was not read to the Group 1 subjects: "During this second part of the experiment, you will again be presented some tones through the headset. (You do not. however, have to worry about receiving an electric shock during this part of the experiment.) Again, please sit quietly; relax but do not fall asleep. Please move as little as possible since movement interferes with our physiological recording."

The "dummy" shock electrode was then removed from the wrist of all subjects. A 15-min rest period was then given, after which Part II of the experiment was administered.

\section{Data Reduction and Analysis}

Phasic skin-resistance response measures were transformed to units of $\log$ conductance using the formula: $\log \left(1 / R_{1}\right)-\log \left(1 / R_{2}\right)$, where $R_{1}$ is the maximum resistance deflection peak occurring within $5 \mathrm{sec}$ of stimulus onset, and $\mathbf{R}_{\mathbf{2}}$ is the prestimulus resistance base level. Tonic skin-resistance levels were measured prior to the onset of each stimulus and converted to conductance units.

The skin-resistance data were measured by scorers who had no knowledge of the experimental contingencies of the present study. The data were punched on computer cards for computation of the above-described transformations and later statistical analyses on an IBM 370 computer.

\section{RESULTS}

Figure 1 plots the mean tonic skin conductance levels for groups over trials, for Parts I and II. They are plotted in blocks of three trials. This figure shows that the group which received the threat of shock instructions (Group 2) is associated with a higher

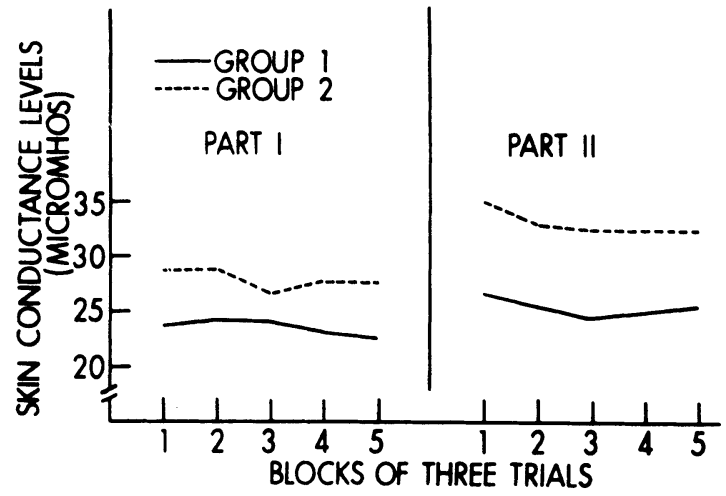

Figure 1. Average skin-conductance levels for groups over trials.

tonic skin conductance level in both Parts I and II, relative to the nonthreatened Group 1 subjects. Statistical analyses confirmed these results. In Part I, a Mann-Whitney test revealed a significant difference between the two groups in the mean skin conductance level across trials, $z=3.01, p<.01$. A MannWhitney test also indicated that these group differences were significant during Part II, $z=2.95$, $\mathrm{p}<.01$.

Average phasic skin conductance response curves for groups, plotted in blocks of three trials, are presented in Figure 2. This figure shows that the Group 2 shock-threatened subjects demonstrated a slower rate of habituation during Part I, relative to the Group 1 subjects. Both groups reached a comparable level of habituation by the end of Part I. However, in Part II, Group 2 demonstrated initially less habituation than Group 1. One between- (groups) and one within- (trials) subjects analysis of variance confirmed these results. In Part I, there was a significant trials effect, $F(14,364)=7.65, p<.001$, indicating the occurrence of habituation over trials. More importantly, there was a significant Group by Trials interaction effect, $F(14,364)=10.83, p<.001$, which statistically confirms that the rate of habituation was slower for the Group 2 subjects. In

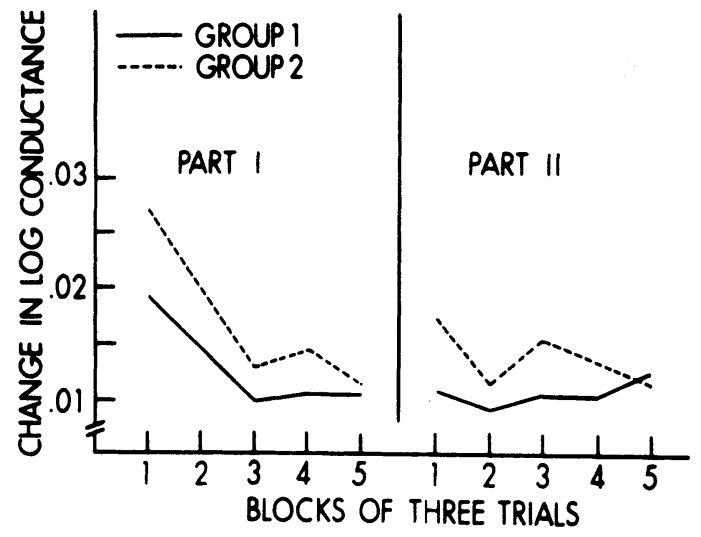
trials. 
Part II, there were again significant trials, $\mathrm{F}(14,364)=3.39, \mathrm{p}<.001$, and Group by Trials, $F(14,364)=2.19, p<.01$, effects. The Group by Trials interaction indicates that Group 2 demonstrated greater responding at the start of Part II, i.e., less long-term habituation.

\section{DISCUSSION}

The results of the present experiment demonstrate that arousal level significantly affects short- and long-term habituation of the OR. In Part I, subjects who received threat of shock instructions displayed significantly higher tonic skin conductance levels, relative to the nonthreatened group. These higher tonic levels were also maintained during Part II of the experiment, even though threat of shock was no longer present. Paralleling these skin conductance level differences, there were significant group differences in the rate of phasic electrodermal response habituation. The shock-threatened group demonstrated a slower rate of short-term habituation. Moreover, even though both groups achieved a comparable level of habituation by the end of Part I, the shock-threatened subjects demonstrated less habituation when the two groups were tested $15 \mathrm{~min}$ later for long-term habituation.. Thus, these data indicate that elevated arousal level produces a decrease in both short- and long-term habituation.

Similar to the short-term habituation results of the present study, Maltzman, Smith, Kantor, and Mandell (1971) had earlier reported that habituation of the electrodermal component of the OR is influenced by the tonic state of the individual. These investigators examined the rate of habituation during stress and nonstress conditions. One stress condition employed, an impending academic examination, has been found to increase tonic levels of autonomic activity (Smith \& Wenger, 1965). Although Maltzman et al. (1971) found no statistically significant differences in tonic skin conductance levels between conditions, there was a clear trend of higher tonic levels in the stress condition, relative to the nonstress condition. More importantly, however, was the finding that the rate of habituation was significantly slower during the stress condition. More recently, Crider and Augenbraun (1975) have also implicated the importance of underlying differences in arousal state as a factor affecting the rate of short-term habituation.

As suggested in an earlier study (Gatchel, 1975), the higher level of physiological arousal may interfere with the consolidation process that prompts habituation. Indeed, the dual-process theory advocated by Groves and Thompson (1970) assumes that habituation is a consequence of both phasic response and state processes. The results of the present investigation clearly demonstrate the importance of state processes, as indexed by sympathetic arousal level, in both short-term and long-term habituation.

\section{REFERENCES}

Crider, A., \& Augenbraun, C. B. Auditory vigilance correlates of electrodermal response habituation speed. Psychophysiology, 1975, 12, 36-40.

DAvis, M. Effects of interstimulus interval length and variability on startle response habituation in the rat. Journal of Comparative and Physiological Psychology, 1970, 72. 177-192.

Gatchel, R. J. Effects of interstimulus interval length on short- and long-term habituation of autonomic components of the orienting response. Physiological Psychology, 1975, 3, 133-136.

Groves, P. M., \& Thompson, R. F. Habituation: A dualprocess theory. Psychological Review, 1970, 77, 419-450.

Maltzman, I., Smith, M. J., Kantor, W., \& Mandell, M. P. Effects of stress on habituation of the orienting reflex. Journal of Experimental Psychology, 1971, 87, 207-214.

SMIth, D. B., \& Wenger, M. A. Changes in autonomic balance during phasic anxiety. Psychophysiology, 1965, 1, 267-271.

(Received for publication July 28, 1975; accepted August 11, 1975.) 